\title{
Endogenous glucose production increases in response to metformin treatment in the glycogen-depleted state in humans: a randomised trial
}

\author{
Mette Marie H. Christensen ${ }^{1}$ • Kurt Højlund ${ }^{2,3}$ • Ole Hother-Nielsen ${ }^{2}$. \\ Tore B. Stage ${ }^{1} \cdot$ Per Damkier $^{1,4} \cdot$ Henning Beck-Nielsen ${ }^{2} \cdot$ Kim Brosen $^{1}$
}

Received: 6 May 2015 / Accepted: 28 July 2015 / Published online: 14 August 2015

(C) Springer-Verlag Berlin Heidelberg 2015

\begin{abstract}
Aims/hypothesis Metformin is believed to reduce glucose levels primarily by inhibiting hepatic glucose production. Recent data indicate that metformin antagonises glucagondependent glucose output, suggesting that compensatory mechanisms protect against hypoglycaemia. Here, we examined the effect of metformin on glucose metabolism in humans after a glycogen-depleting fast and the role of reducedfunction alleles in $O C T 1$ (also known as SLC22A1).

Methods In a randomised, crossover trial, healthy individuals with or without reduced-function alleles in $O C T 1$ were fasted for $42 \mathrm{~h}$ twice, either with or without prior treatment with $1 \mathrm{~g}$ metformin twice daily. Participants were recruited from the Pharmacogenomics Biobank of the University of Southern Denmark. Treatment allocation was generated by the Good Clinical Practice Unit, Odense University Hospital, Denmark. Variables of whole-body glucose metabolism were assessed using $\left[3-{ }^{3} \mathrm{H}\right] \mathrm{glucose}$, indirect calorimetry and
\end{abstract}

Electronic supplementary material The online version of this article (doi:10.1007/s00125-015-3733-2) contains peer-reviewed but unedited supplementary material, which is available to authorised users.

Mette Marie H. Christensen

mmchristensen@health.sdu.dk

1 Department of Public Health, Clinical Pharmacology, University of Southern Denmark, J.B. Winsloews Vej 19, 5000 Odense, Denmark

2 Department of Endocrinology, Odense University Hospital, Odense, Denmark

3 Section of Molecular Diabetes \& Metabolism, Institute of Molecular Medicine \& Institute of Clinical Research, University of Southern Denmark, Odense, Denmark

4 Department of Clinical Chemistry and Pharmacology, Odense University Hospital, Odense, Denmark measurement of substrates and counter-regulatory hormones. The primary outcome was endogenous glucose production (EGP).

Results Thirty-seven individuals were randomised. Thirty-four completed the study (12 had none, 13 had one and nine had two reduced-function alleles in $O C T 1$ ). Three were excluded from the analysis because of early dropout. Metformin significantly stimulated glucose disposal rates and non-oxidative glucose metabolism with no effect on glucose oxidation. This increase in glucose utilisation was explained by a concomitant increase in glycolytic flux and accompanied by increased EGP, most likely mediated by increased plasma lactate, glucagon and cortisol levels. There was no effect of reduced-function $O C T 1$ alleles on any of these measures. All individuals completed the glycogendepleting fast without hypoglycaemia.

Conclusions/interpretation Metformin stimulates glycolytic glucose utilisation and lactate production in the glycogendepleted state. This may trigger a rise in glucose counterregulatory hormones and subsequently an increase in EGP, which protects against hypoglycaemia.

Trial registration: ClinicalTrials.gov NCT01400191

Funding: Danish Research Council for Health and Disease (0602-02695B) and Odense University Hospital Free Research Fund, 2012.

Keywords EGP $\cdot$ Fast $\cdot$ Metformin $\cdot$ OCT1
Abbreviations
EGP Endogenous glucose production
HGP Hepatic glucose production
mGPD Mitochondrial glycerophosphate dehydrogenase
NOGM Non-oxidative glucose metabolism
OCT1/2 Organic cation transporter $1 / 2$ 
$\mathrm{Ra} \quad$ Rate of glucose appearance

Rd Rate of glucose disposal

REE Resting energy expenditure

RER Respiratory exchange ratio

SNP Single nucleotide polymorphism

\section{Introduction}

The oral anti-hyperglycaemic agent metformin is a cornerstone in the treatment of type 2 diabetes [1]. It is believed to reduce glucose levels mainly by decreasing hepatic glucose production (HGP) in type 2 diabetic patients through inhibition of gluconeogenesis [2], and an augmentation of the peripheral glucose uptake and utilisation [3]. The latter may involve an increased anaerobic glucose metabolism in the intestine owing to accumulation of metformin in this tissue [4]. Metformin lowers plasma glucose levels and $\mathrm{HbA}_{1 \mathrm{c}}$ in a doserelated manner [5], and unlike other glucose-lowering agents such as sulfonylureas and insulin, this effect is achieved without weight gain or hypoglycaemia.

At the cellular level, the mode of action of metformin has been debated for several years. Inhibition of the mitochondrial respiratory chain complex 1 has been suggested as a primary molecular target [6]. Inhibition of mitochondrial respiration decreases the cellular energy charge. The concomitant increase in AMP levels activates AMP-activated protein kinase (AMPK), which leads to inhibition of glucose production by reducing the expression of gluconeogenic enzymes [6], but also inhibits adenylate cyclase causing reduced intracellular levels of cAMP [7]. The latter has been shown to abrogate activation of protein kinase $\mathrm{A}$ and by this mechanism antagonise glucagon-dependent glucose output in hepatocytes in mice [7]. Most recently, it was reported that metformin suppresses gluconeogenesis by inhibiting the hepatic redox shuttle enzyme mitochondrial glycerophosphate dehydrogenase (mGPD) in rodents [8]. Thus, metformin indirectly decreases the substrate fluxes of lactate and glycerol to the gluconeogenesis and the flux of electrons to the mitochondrial respiratory chain [8]. It remains to be elucidated to what extent metformin continues to block HGP by these mechanisms under conditions in which glucose counter-regulatory mechanisms are activated to avoid hypoglycaemia.

Metformin is an organic cation and its passage across cell membranes depends on solute carrier transporters. The intestinal uptake of metformin is mediated by proton-activated plasma membrane monoamine transporter (PMAT) and organic cation transporter 1 (OCT1) [9, 10]. The hepatic uptake of metformin is predominantly facilitated by OCT1. Metformin is not metabolised and the entire absorbed drug is excreted into urine. The organic cation transporter 2 (OCT2), multidrug and toxin extrusion transporters 1 (MATE1) and 2-K (MATE2-K) facilitate the renal transcellular transport [11]. Genetic variation in all of the above-mentioned transporters has been linked to altered pharmacokinetic and pharmacodynamic response to metformin [9]. In OCT1 (also known as SLC22A1), there are four known genetic variations (rs12208357 [R61C], rs34130495 [G401S], rs34059508 [G465R] and rs72552763 [M420del]), which encode an OCT1-transporter protein with reduced function and hence, have been linked to reduced-response metformin response in humans [12-14].

The main purpose of the present study was to evaluate the effect of metformin on endogenous glucose production (EGP), i.e. on gluconeogenesis in the glycogen-depleted state in fasting healthy individuals with or without reducedfunction OCT1 diplotypes (none, one or two minor alleles). Our hypothesis was that individuals with reduced-function OCT1 diplotypes have a decreased transport of metformin into the hepatocytes and a diminished response on HGP compared with individuals with only wild-type alleles. Moreover, we examined the hypothesis that glucose counter-regulatory hormones or other compensatory mechanisms are activated to prevent hypoglycaemia in response to treatment with metformin during prolonged fast. To our knowledge, this is the first study to evaluate the pharmacodynamics of metformin at steady state during repeated dosing in fasting healthy individuals in relation to different $O C T 1$ genotypes.

\section{Methods}

\section{Study participants}

From the Pharmacogenomics Biobank of the University of Southern Denmark, healthy individuals were, in 2012, included based on their OCT1 genotype [15]. All individuals were healthy with renal and hepatic functions within normal range as assessed by plasma creatinine and plasma aminotransferase. None had a history of alcohol abuse or took any medication, and all gave written informed consent to participate in the study. The genotypes of OCT1, OCT2 (also known as SLC22A2), MATE1 (also known as SLC47A1) and MATE2-K (also known as $S L C 47 A 2)$ including the haplotypes and diplotypes of the reduced-function alleles in OCT1 have previously been described [16].

\section{Study design}

The study was designed as a randomised crossover trial with a wash-out period of at least 4 weeks between the phases. Sealed envelopes were used for the randomisation procedure. The random allocation sequence was generated with assistance from the Good Clinical Practice (GCP) unit, Odense University Hospital, Denmark. The primary investigator enrolled the participants. To ensure that gluconeogenesis was the 
main contributor to glucose production, the healthy individuals were fasted for $42 \mathrm{~h}$, after which hepatic glycogen stores are known to be almost completely exhausted [17]. Moreover, the liver is the main gluconeogenic source when individuals are fasted for $<60 \mathrm{~h}$. However, as time goes by, the contribution from the kidneys will increase [17].

Phase A The individuals were admitted to the Department of Endocrinology, Odense University Hospital, Denmark, at 16:00 hours after $20 \mathrm{~h}$ of fast. The next day at 08:00 hours, after $36 \mathrm{~h}$ of fast, the experiment was initiated. Two catheters were inserted into contralateral antecubital veins. One of them was used for $\left[3-{ }^{3} \mathrm{H}\right]$ glucose tracer infusion and the other for collection of blood samples. The latter was placed and maintained in a heated plexiglass box for arterialisation of venous blood. A primed-constant intravenous infusion of $\left[3-{ }^{3} \mathrm{H}\right]$ glucose was initiated and continued throughout the next $6 \mathrm{~h}$ using a precision syringe pump (Harvard Apparatus, Natick, MA, USA). The ratio between priming dose and constant tracer infusion was 100:1. To achieve a common level of basal plasma-specific activity, the tracer infusion rate was adjusted for body surface area by adjustment of the infusate volume as previously described $[18,19]$. Blood samples were collected at timed intervals $(0,1,2,3,4,5,5.5$ and $6 \mathrm{~h})$ for determination of plasma cortisol, plasma NEFA, plasma glucagon, serum insulin and C-peptide. For plasma glucose, plasma lactate and plasma $\left[3-{ }^{3} \mathrm{H}\right]$ glucose activity, blood samples were collected every $15 \mathrm{~min}$ for the $6 \mathrm{~h}$ period. Both plasma glucose and lactate were measured bedside. Additional blood samples were immediately centrifuged; serum samples were stored at $-80^{\circ} \mathrm{C}$ and plasma samples at $-20^{\circ} \mathrm{C}$ until analysis. Indirect calorimetry was performed using a ParvoMedics TrueOne 2400 (Sandy, UT, USA) automated gas analysis system. After an equilibration period of $10 \mathrm{~min}$, the average gas exchange rates recorded over the two 30-min steady-state periods (90-120 min and 330-360 min) were used to calculate rates of glucose oxidation, lipid oxidation, and respiratory exchange ratio (RER) and resting energy expenditure (REE) as previously described [20]. The protein oxidation rate was estimated from urinary urea nitrogen excretion $(1 \mathrm{~g}$ nitrogen $=6.25 \mathrm{~g}$ protein $)$ and corrected for changes in pool size.

Phase B When the experiment and the sampling started in this phase, all individuals were in steady state with 1,000 mg metformin twice daily. For 7 days, they had ingested tablets of metformin (Metformin 'Actavis', Denmark, $500 \mathrm{mg}$ ) at 08:00 hours and 20:00 hours (Day 1: $500 \mathrm{mg}$ a.m. and $500 \mathrm{mg}$ p.m.; Day 2: $500 \mathrm{mg}$ a.m., 1,000 mg p.m.; Days 3, 4, 5 and 6: 1,000 mg a.m. and 1,000 mg p.m.; Day 7: 1,000 mg a.m.). As in phase $\mathrm{A}$, the individuals were admitted to the Department of Endocrinology, Odense University Hospital, Denmark, at 16:00 hours after $20 \mathrm{~h}$ of fast. The experiment was initiated the next day at 08:00 hours, after $36 \mathrm{~h}$ of fast.
The experiment was performed as in phase A and the fast likewise ended after $42 \mathrm{~h}$.

\section{Study procedure}

The study was conducted in accordance with the Helsinki Declaration and Good Clinical Practice and monitored by the GCP unit, Odense University Hospital, Odense, Denmark. It was approved by the Danish Health and Medicines Authority (J. no: 2011050747), the Danish Data Protection Agency (J. no. 2011-41-6231) and the Regional Committee on Biomedical Research Ethics of Southern Denmark (Project ID: S-20110082). It is registered in the European Clinical Trial Database (EudraCT no.: 2011001696-39) and at ClinicalTrials.gov (registration no. NCT01400191).

\section{Analytical methods}

Plasma glucose and lactate were measured using an ABL800 FLEX Analyzer (Radiometer, Copenhagen, Denmark). Serum insulin and C-peptide were measured using COBAS immunoassay platforms (Roche Diagnostics, IN, USA). Plasma NEFA concentration was measured using a colorimetric assay kit (Wako Chemicals, Richmond, VA, USA) using a COBAS FARA 2 Autoanalyzer (Roche Diagnostic, Rotkreuz, Switzerland). Serum cortisol was measured by a chemiluminescent method using an Immulite 2000 (DPC Cirrus, Los Angeles, CA, USA). The plasma glucagon concentration was determined by a validated antibody method previously described [21].

\section{Statistical analysis and considerations}

The demographic data are presented as medians with 25th to 75th percentiles; others are listed as means with $95 \%$ CIs unless otherwise specified. The difference in the EGP was, guided by qq-plots, found to follow a Gaussian distribution. Statistical inference of the primary endpoint for OCT1 diplotypes was analysed using one-way ANOVA. Paired $t$ tests were used to determine significance between the two periods. A level of $p<0.05$ was considered statistically significant. All statistical analyses were performed using STATA 11.0 (StataCorp, College Station, TX, USA).

\section{Sample size}

The sample size calculation was based on the primary outcome represented by differences in HGP between individuals homozygous and heterozygous for reduced-function alleles in OCT1 (rs12208357, rs72552763, rs34130495 and rs34059508). Based on an interindividual coefficient of variance for HGP of $18 \%$ after $40 \mathrm{~h}$ of fast [22], it was estimated that a true difference of $25 \%$ could be detected, given a two- 
sided level of significance of 0.05 and a power of $80 \%$, by using 12 individuals in each group and taking into account a dropout rate of $20 \%$.

\section{Calculations}

After a $2 \mathrm{~h}$ basal tracer equilibration period, calculation of glucose turnover rates for the following $4 \mathrm{~h}$ period was based on determinations of plasma glucose concentration and $\left[3-{ }^{3} \mathrm{H}\right]$ glucose activity as previously described [18]. Rates of total glucose appearance $(\mathrm{Ra})$ and glucose disposal $(\mathrm{Rd})$ were calculated using Steele's non-steady-state equations [18]. In these calculations, the distribution volume of glucose was set to $200 \mathrm{ml} / \mathrm{kg}$ body weight and the pool fraction to 0.65 [18]. In the final $4 \mathrm{~h}$ period, EGP was assumed to equal Ra. The in vivo glycolytic flux rates were calculated from the generation rates of plasma ${ }^{3} \mathrm{H}_{2} \mathrm{O}$ from $\left[3-{ }^{3} \mathrm{H}\right]$ glucose, assuming that all tritium in the $\mathrm{C}-3$ position was lost to water during the glycolytic process as previously described in detail [23]. Non-oxidative glucose metabolism (NOGM) was calculated as the difference between $\mathrm{Rd}$ and glucose oxidation.

\section{Genotyping, linkage disequilibrium, haplotype and diplotype inference}

Genotyping of OCT1 including inferring the haplotypes and diplotypes of the reduced-function alleles in $O C T 1$ has previously been described in detail in a separate publication [16].

\section{Results}

Thirty-seven healthy individuals of European descent participated in the study, of which 34 (12 women and 22 men) completed the study. One was excluded due to early onset of diarrhoea and two found the fast too challenging. Twelve had none, 13 had one, and nine had two reduced-function alleles in OCT1. The flow diagram is shown in Electronic Supplementary Material (ESM) Fig. 1. The demographic characterisation is shown in Table 1. At inclusion, no significant differences in the values were seen among the different $O C T 1$ diplotypes. The distribution of sex for 0,1 or 2 reduced-function $O C T 1$ alleles was (men/women) 7/5, 9/4 and 6/3.

During treatment with metformin, both EGP and Rd increased significantly in the study period from 2 to $6 \mathrm{~h}$ (38-42 h fast): (EGP control vs EGP $_{\text {metformin }}, 56.2 \mathrm{vs}$ $70.1 \mathrm{mg} \mathrm{min}{ }^{-1} \mathrm{~m}^{-2} ; \mathrm{Rd}_{\text {control }} \mathrm{vs} \mathrm{Rd}_{\text {metformin }}, 57.3 \mathrm{vs}$ $70.6 \mathrm{mg} \mathrm{min}^{-1} \mathrm{~m}^{-2}$ [Table 2, Fig. 1]). Moreover, in response to treatment with metformin, both the NOGM and the glycolytic flux increased significantly during the 30-min steady-state period from 330 to $360 \mathrm{~min}$ (41.5-42 h fast): (NOGM ${ }_{\text {control }}$ Vs $\mathrm{NOGM}_{\text {metformin }}, 49.6$ vs $64.9 \mathrm{mg} \mathrm{min}{ }^{-1} \mathrm{~m}^{-2}$; Glycolytic flux $x_{\text {control }}$ vs Glycolytic flux metformin, 43.9 vs
Table 1 Demographic information at inclusion

\begin{tabular}{lll}
\hline Demographic information & Median & 25th-75th percentile \\
\hline Age at inclusion (years) & 25 & $24-27$ \\
$\mathrm{HbA}_{1 \mathrm{c}}(\%)$ & 5.1 & $5.0-5.3$ \\
$\mathrm{HbA}_{\mathrm{lc}}(\mathrm{mmol} / \mathrm{mol})$ & 32 & $31-34$ \\
$\mathrm{BMI}\left(\mathrm{kg} / \mathrm{m}^{2}\right)$ & 23.0 & $22.2-25.2$ \\
$\mathrm{BSA}\left(\mathrm{m}^{2}\right)$ & 2.0 & $1.8-2.1$ \\
Plasma creatinine $(\mu \mathrm{mol} / \mathrm{l})$ & 80 & $71-85$ \\
Plasma alanine aminotransferase $(\mathrm{U} / \mathrm{l})$ & 24 & $20-32$ \\
HDL-cholesterol (mmol/l) & 1.4 & $1.2-1.7$ \\
LDL-cholesterol $(\mathrm{mmol} / \mathrm{l})$ & 2.9 & $2.4-3.3$ \\
Triacylglycerol $(\mathrm{mmol} / \mathrm{l})$ & 1.0 & $0.8-1.3$ \\
Total cholesterol $(\mathrm{mmol} / \mathrm{l})$ & 4.7 & $4.3-5.6$ \\
GFR $_{\mathrm{i}}(\mathrm{ml} / \mathrm{min})$ & 108 & $91-120$ \\
\hline
\end{tabular}

BSA, Body surface area $=$ Weight $^{0.425} \times$ Height $^{0.725} \times 0.007184$ [40]

GFR $_{\mathrm{i}}$ : The individual GFRs $=(\mathrm{eGFR} \times \mathrm{BSA}) / 1.73 \mathrm{~m}^{2}=[175 \times($ plasma creatinine $/ 88.4)^{-1.154} \times(\text { age })^{-0.203} \times(0.742$ if female $) \times(1.21$ if AfricanAmerican $) \times \mathrm{BSA}] / 1.73 \mathrm{~m}^{2}[41]$

$57.2 \mathrm{mg} \mathrm{min}^{-1} \mathrm{~m}^{-2}$ [Table 3]). No significant changes were seen in RER, REE, glucose or lipid oxidation. Consistent with the steady decline in plasma glucose from 2 to $6 \mathrm{~h}$, Rd was slightly higher than EGP both during the control (difference $1.1 \mathrm{mg} \mathrm{min}^{-1} \mathrm{~m}^{-2}[95 \%$ CI $0.8,1.3] ; p=1.2 \times 10^{-8}$ ) and the metformin (difference $0.5 \mathrm{mg} \mathrm{min}^{-1} \mathrm{~m}^{-2}$ [95\% CI 0.2, 1.0]; $p=$ 0.007) period.

Plasma glucose, insulin, C-peptide and NEFA mean values did not differ statistically; however, during the first 2-3 h (36-39 $\mathrm{h}$ fast), the plasma glucose levels visually appeared to be lower during metformin treatment (Figs 1, 2). Mean plasma lactate increased significantly during metformin treatment; however, it remained within the normal range $(<2.0 \mathrm{mmol} / \mathrm{l})$ : Plasma lactate control $_{\text {vs plasma lactate }}$ metformin, $0.85 \mathrm{vs} 0.95 \mathrm{mmol} / \mathrm{l}$. Both plasma cortisol and glucagon mean values increased significantly when the healthy individuals were treated with metformin: (plasma cortisol $\mathrm{c}_{\text {control }}$ vs plasma cortisol $_{\text {metformin }}, 367$ vs $425 \mathrm{nmol} / 1$; plasma glucagon control $_{\text {vs }}$ plasma glucagon $_{\text {metformin }}, 12.8 \mathrm{vs} 14.2 \mathrm{pmol} / 1$ [Table 2, Figs 1 , 2]). As observed previously at the end of a $72 \mathrm{~h}$ fast [24], we observed higher levels of all measured substrates and hormones in the beginning of the study period after the first $36 \mathrm{~h}$ of fasting. These baseline samples were drawn before any infusion or priming dose was given. This could be explained by the anticipation of food intake (the cephalic phase) as well as peak levels of cortisol in the morning [25].

The different $O C T 1$ diplotypes had no effect on EGP, Rd, glycolytic flux, substrate oxidation, NOGM or plasma/serum levels of glucose, lactate, insulin, C-peptide, cortisol or NEFA (ESM Tables 1, 2). For plasma glucagon, there was a significant difference for the different $O C T 1$ diplotypes: none, one or two reduced-function alleles: $2.4,-0.8$ and $1.8 \mathrm{pmol} / \mathrm{l}$; 
Table 2 The impact of metformin on the endogenous glucose turnover, substrates and hormones during a glycogen-depleting fast

\begin{tabular}{llll}
\hline Mean $_{2-6 \mathrm{~h}}$ & Control & Metformin treatment & $p$ \\
\hline EGP $\left(\mathrm{mg} \mathrm{min}^{-1} \mathrm{~m}^{-2}\right)$ & $56.2(54.4,58.1)$ & $70.1(67.6,72.6)$ & $3 \times 10^{-13}$ \\
Rd $\left(\mathrm{mg} \mathrm{min}^{-1} \mathrm{~m}^{-2}\right)$ & $57.3(55.5,59.1)$ & $70.6(68.2,73.1)$ & $8 \times 10^{-13}$ \\
Plasma glucose $(\mathrm{mmol} / \mathrm{l})$ & $3.7(3.6,3.9)$ & $3.7(3.5,3.9)$ & 0.52 \\
Plasma lactate $(\mathrm{mmol} / \mathrm{l})$ & $0.85(0.80,0.89)$ & $0.95(0.90,1.00)$ & $4 \times 10^{-5}$ \\
Serum insulin $(\mathrm{pmol} / \mathrm{l})$ & $14.8(12.8,16.7)$ & $16.1(13.9,18.3)$ & 0.14 \\
Serum C-peptide $(\mathrm{pmol} / \mathrm{l})$ & $223(202,244)$ & $221(197,244)$ & 0.77 \\
Serum cortisol $(\mathrm{nmol} / \mathrm{l})$ & $367(320,414)$ & $425(370,480)$ & 0.002 \\
Plasma glucagon $(\mathrm{pmol} / \mathrm{l})$ & $12.8(11.1,14.5)$ & $14.2(12.5,16.0)$ & $0.03^{\mathrm{a}}$ \\
Plasma NEFA $(\mu \mathrm{mol} / \mathrm{l})$ & $1,194(1,101,1,286)$ & $1,205(1,112,1,298)$ & 0.56 \\
\hline
\end{tabular}

Data are presented as means with $95 \%$ CIs

Statistical inferences of the results were analysed using a paired $t$ test

${ }^{a}$ Owing to the visual inclining response, statistical inference $\mathrm{AUC}_{2-6}$ for glucagon was analysed using a paired $t$ test. Glucagon mean is estimated as: $\mathrm{Mean}_{2-6}=\left(\mathrm{AUC}_{2-6}\right) / 4 \mathrm{~h}$

$p=0.02$. However, the observation is ambiguous. No significant changes were seen in RER or REE between the periods for any of the OCT1 diplotypes (data not shown). When tested individually, none of the single nucleotide polymorphisms
(SNPs) in OCT1 (rs12208357, rs34130495, rs72552763, rs34059508, rs461473, rs622342), OCT2 (rs316019), MATE1 (rs2289669, rs2252281) or MATE2-K (rs12943590) significantly affected the observed increase in EGP; nor were
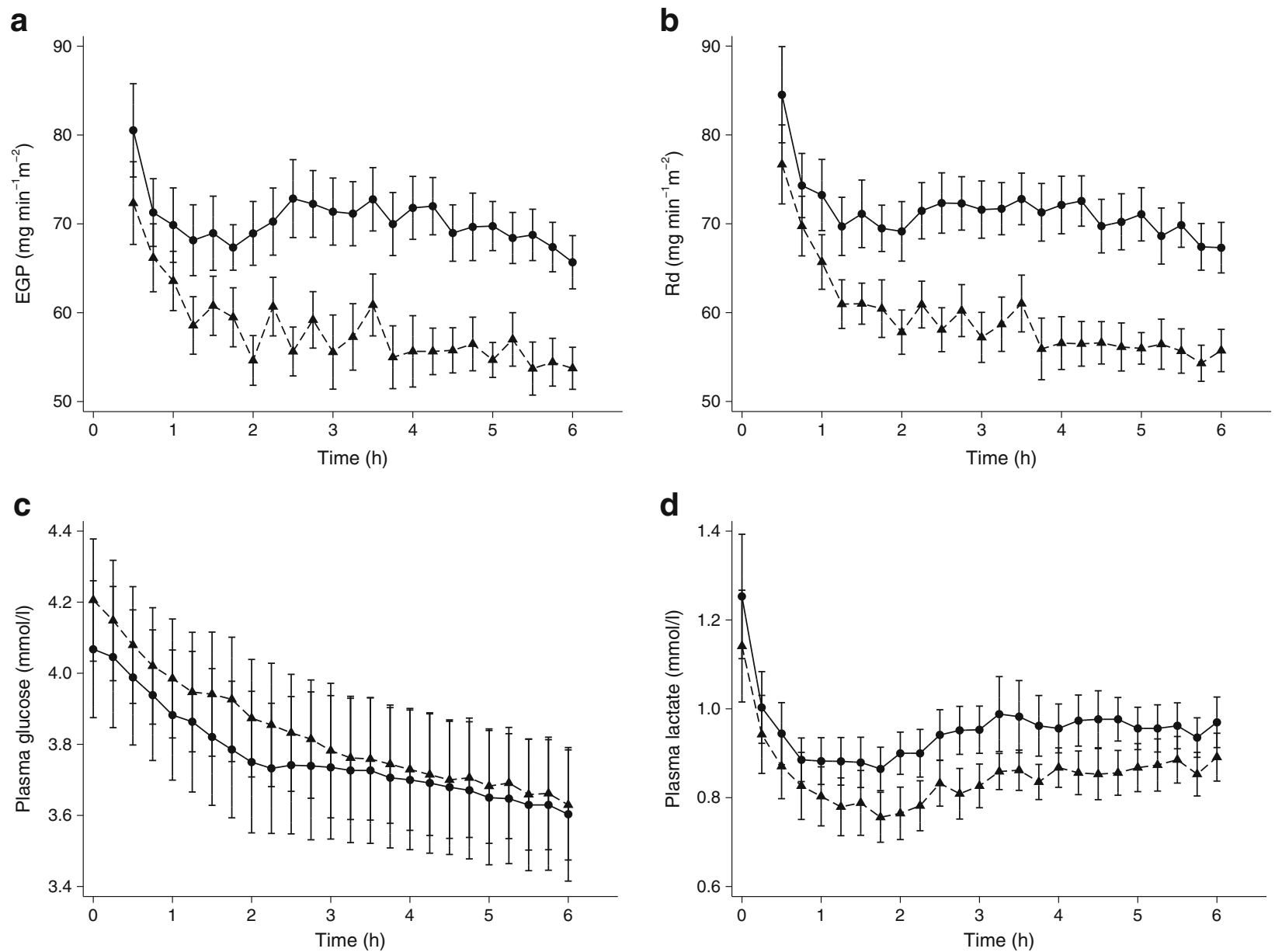

Fig. 1 Graphic illustrations of EGP (a), Rd (b), plasma glucose (c) and plasma lactate (d) profiles for the two study periods. Data are presented as means with $95 \%$ CIs. Circles, metformin period; triangles, control period 
Table 3 The impact of metformin on NOGM, glucose oxidation, lipid oxidation and the glycolytic flux

\begin{tabular}{llll}
\hline Mean $_{5.5-6 \mathrm{~h}}$ & Control & Metformin treatment & $p$ \\
\hline NOGM & $49.6(42.2,57.0)$ & $64.9(60.5,69.2)$ & 0.001 \\
Glucose oxidation & $5.1(-2.9,13.1)$ & $2.9(-1.6,7.3)$ & 0.61 \\
Lipid oxidation & $45.3(39.9,50.6)$ & $47.0(42.7,51.2)$ & 0.57 \\
Glycolytic flux & $43.9(42.1,45.7)$ & $57.2(54.9,59.0)$ & $1.7 \times 10^{-11}$ \\
RER & $0.74(0.73,0.75)$ & $0.73(0.72,0.74)$ & 0.14 \\
REE (kJ/day) & $6,863(6,362,7,364)$ & $6,978(6,541,7,415)$ & 0.62 \\
\hline
\end{tabular}

All the rates of metabolism are given as $\mathrm{mg} \mathrm{min}^{-1} \mathrm{~m}^{-2}$

The results are presented as means with $95 \%$ CIs

Indirect calorimetry was performed over the 30-min steady-state period from 330 to $360 \mathrm{~min}$

Statistical inferences of the Mean $_{5.5-6} \mathrm{~h}$ result were analysed using a paired $t$ test

any interactions between rs2252281 and rs12943590 or rs2289669 and rs622342 observed.

\section{Discussion}

In this study, we examined the effect of metformin on wholebody glucose metabolism in healthy individuals after a glycogen-depleting fast and the role of reduced-function alleles in OCT1. All individuals completed both of the glycogen-depleting fasting periods without hypoglycaemia. We found that metformin significantly stimulated glucose disposal rates, NOGM, glycolytic flux and increased plasma lactate, glucagon and cortisol levels. This was accompanied by a comparable increase in EGP, whereas no effect on glucose and lipid oxidation or plasma glucose, insulin, C-peptide and NEFA levels was observed. In contrast to our hypothesis, we found no effect of the reduced-function $O C T 1$ alleles for any of the measured variables. Our data support the notion that metformin stimulates glycolytic glucose utilisation, and that the ability of metformin to inhibit hepatic glucose output can be overruled by a rise in counter-regulatory hormones to protect against hypoglycaemia.

Evidence from clinical studies suggests that a pivotal function of metformin is to inhibit gluconeogenesis and thus decrease HGP in patients with type 2 diabetes [2,26]. The basal rate of glucose production and of gluconeogenesis has been reported to be higher in patients with type 2 diabetes compared with healthy individuals [2]. In the present study, it was therefore to some extent unexpected that EGP increased significantly when the fasting healthy individuals were treated with metformin. It appeared that this increase in EGP was a counterregulatory response to the observed stimulation of glycolytic glucose utilisation and lactate production, including increasing levels of the glucose counter-regulatory hormones, glucagon and cortisol. This interpretation is supported by evidence that metformin accumulates in the intestine and stimulates anaerobic glucose metabolism to lactate [4]. Moreover, to our knowledge, there is no evidence that metformin directly stimulates EGP or that an increased EGP per se is capable of driving an increased glycolytic flux. Thus, under the given study conditions, it is reasonable to assume that the glycogen-depleting fast combined with the metformin-induced peripheral glucose utilisation caused a rise in EGP rather than the reverse to protect against hypoglycaemia. Although further studies are warranted, our study suggests that metformin may not inhibit EGP in healthy individuals. However, our study does not exclude that the glucose-lowering effect of metformin in patients with type 2 diabetes is mainly due to inhibition of HGP, e.g. by blocking glucagon-dependent hepatic glucose output $[7,8]$. In patients with type 2 diabetes this could involve increased circulating glucagon levels and enhanced hepatic sensitivity to glucagon, which is believed to contribute to an elevated HGP [27-29]. Furthermore, the counter-regulatory system may not be fully intact in patients with type 2 diabetes, and, therefore, further studies are needed to verify these mechanisms in the target cohort.

When individuals are fasted for $<60 \mathrm{~h}$, the liver is the main gluconeogenic source [17]. Although our study method only allows determination of EGP, this suggests that the observed increase in glucose output in response to metformin was mainly coming from the liver. After $60 \mathrm{~h}$ of fast, renal gluconeogenesis accounts for $\sim 20 \%$ of the endogenous glucose turnover [17]. In addition, animal data indicate that during fasting, intestinal glucose production could be present [30], but this observation needs further validation in humans. However, even if extrahepatic EGP contributes to the regulation of gluconeogenesis during our study conditions, it is most likely that the kidney and intestine would respond similarly to metformin and the observed increased glycolytic flux as the liver. While any extrahepatic EGP may limit our ability to draw conclusions concerning HGP, it would not confound the overall interpretation that EGP is increased to protect against hypoglycaemia.

Our findings are in agreement with what we [3] and others [4] have previously shown, i.e. that metformin augments the 

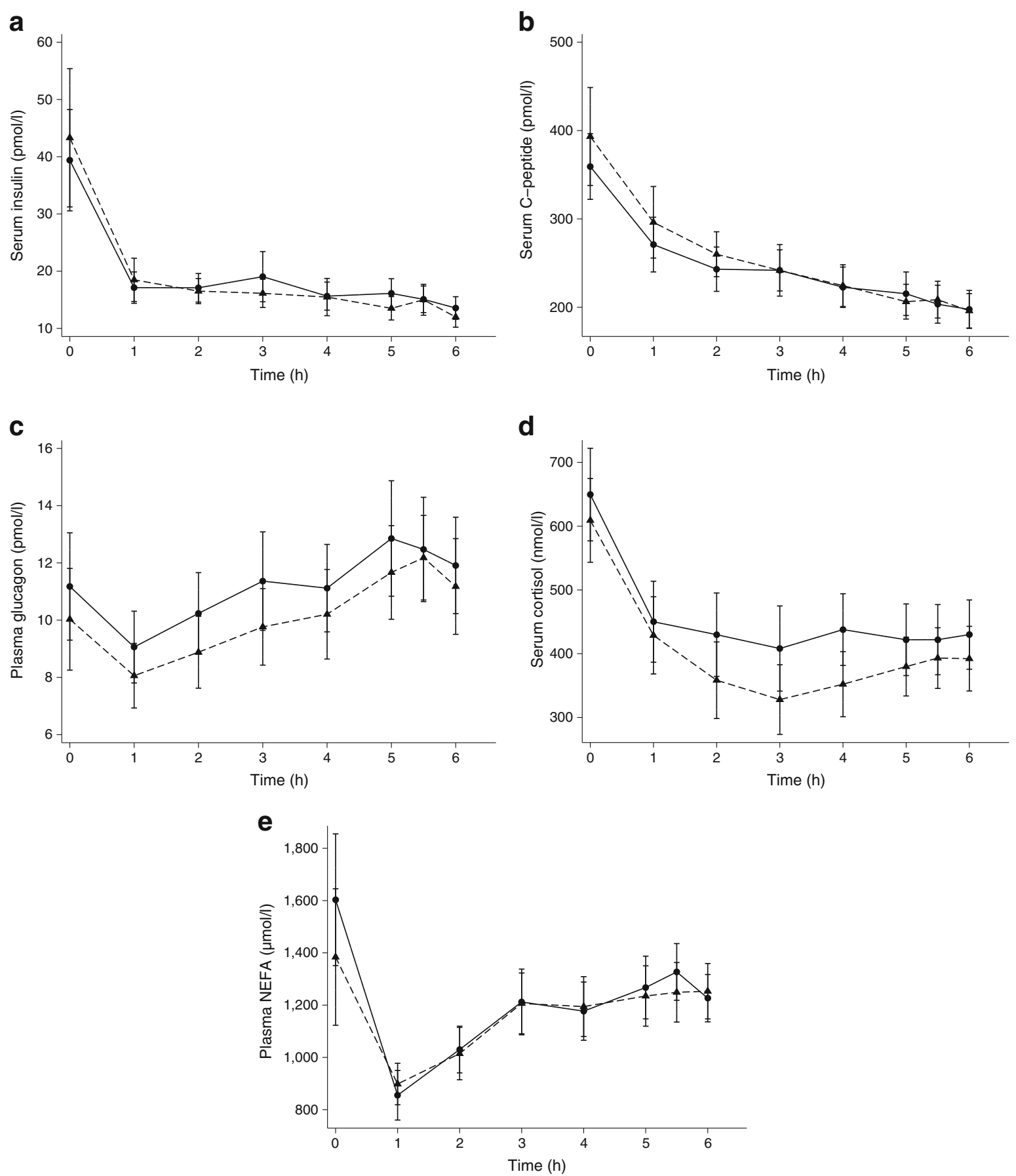

Fig. 2 Graphic illustrations of serum insulin (a), serum C-peptide (b), plasma glucagon (c) serum cortisol (d) and plasma NEFA (e) profiles for the two study periods. Data are presented as means with $95 \%$ CIs. Circles, metformin period; triangles, control period

peripheral glucose uptake. The increase in Rd was fully explained by the observed concomitant increase in glycolytic flux. Our data indicate that to protect against hypoglycaemia, this increase in glycolytic flux was accompanied by an increased EGP that appeared to be mediated by the increased plasma lactate, glucagon and cortisol levels. However, our study is limited by the use of a single tracer approach, and our data do not fully prove that the observed increases in lactate, glucagon and cortisol are responsible for the increase in EGP. This needs to be clarified in further studies.

The NOGM increased significantly during metformin treatment. Generally speaking, NOGM is the result of glycogen storage or lactate production [31]. As a result of the fasting condition, the observed increase in NOGM reflects a nonstorage situation. In agreement with this, we also observed an increased lactate production. Metformin is mainly absorbed 
in the small intestine [32], and it has been shown to accumulate in enterocytes and increase the anaerobic glucose metabolism [4]. A portal surplus of intestinally generated lactate can be extracted by liver and converted back to glucose. It has been speculated that this splanchnic glucose-lactate cycle could sustain gluconeogenesis and protect against metformin-induced hypoglycaemia during fasting [33]. In contrast, metformin has not been shown to increase lactate production in muscle, fat or brain tissue [4]. However, further studies are necessary to establish the potential role of this splanchnic glucose-lactate cycle in response to metformin.

Recently, it was suggested that metformin suppresses gluconeogenesis by inhibiting the redox shuttle enzyme mGPD and indirectly decreases the substrate fluxes of lactate and glycerol to gluconeogenesis in the liver [8]. Others have suggested that metformin indirectly antagonises the major glucose counterregulatory hormone glucagon [7]. If these two proposed mechanisms were essential for metformin's mode of action and completely block the ability to raise HGP, protection against hypoglycaemia would be impaired during fast. Our results convincingly demonstrate that this is not the case in fasting healthy individuals. We find that a surplus of lactate, glucagon and cortisol appears to be able to stabilise plasma glucose and overrule the increased glycolytic flux during metformin treatment. In support, $1 \mathrm{~g}$ metformin given intravenously to healthy individuals does not possess any acute direct hypoglycaemic effect [34]. This observation suggests that intestinal passage with local effects of metformin could be of importance for the response to metformin. However, we cannot exclude that repeated dosing is needed, whether given i.v. or orally, in order to achieve accumulation and stimulation of glycolytic processes in tissues such as enterocytes, for example.

Both the pharmacokinetics and -dynamics of metformin are subject to drug-drug interactions [35], and the steadystate plasma concentration of metformin seems to be under little, if any, genetic control [36] even though the pharmacodynamics is more tightly genetically controlled [37]. In the present study, we also investigated the putative role of $O C T 1$ on both the pharmacokinetics [16] and -dynamics of metfor$\mathrm{min}$. The pharmacological rationale for this approach was that metformin, due to its hydrophilic properties, depends highly on OCT1 membrane transport to reach its hepatic site of action. Individuals carrying reduced-function $O C T 1$ diplotypes were expected to have a decreased transport of metformin into the hepatocytes and a smaller reduction in the HGP compared with individuals carrying wild-type alleles. As described above, neither the EGP nor any of the other variables were affected by the $O C T 1$ allele status. This is in contrast to previous studies $[12,13,38]$ and in agreement with others [14, 39]. Most studies differ with respect to dosing of metformin (not only in amount but also single dose vs steady state) or participant population (type 2 diabetic patients vs healthy individuals) and may not be directly comparable. In the present study, we used EGP as a measure of metformin effect instead of the OGTT previously applied. We believe that the OGTT is a suboptimal test for a pharmacodynamic evaluation of metformin. We report that metformin increases EGP in healthy individuals suggesting that the primary site of action for metformin is not the liver. If indeed metformin stimulates the intestinal glycolytic glucose utilisation in healthy individuals, changes in OGTT in response to metformin treatment may reflect an increased intestinal utilisation of glucose and not a decreased hepatic glucose output.

We cannot fully exclude the possibility that reducedfunction $O C T 1$ variants could play a minor role for the effect of metformin on HGP and/or $\mathrm{HbA}_{1 \mathrm{c}}$ in patients with type 2 diabetes. Prospective geno- to phenotype tracer studies may help answer this question. However, future pharmacogenomics studies with metformin should focus on applying more polygenic approaches instead of focusing on SNPs in few genes.

In summary, we have demonstrated that metformin in the glycogen-depleted fast state stimulates glycolytic glucose utilisation and lactate production. To protect against hypoglycaemia, this appears to trigger a rise in glucose counter-regulatory hormones and subsequently an increase in the EGP. Our findings exclude that differences in EGP of 25\% or more can be related to the $O C T 1$ genomic variants studied. We found no statistically significant differences among the secondary variables, and, therefore, clinically meaningful effects of the $O C T 1$ genotype variants on the pharmacodynamics of metformin appear unlikely. The latter finding suggests that the liver may not be the primary site of action of metformin. While further studies are needed, this study provides important insight into compensatory mechanisms by which hypoglycaemia is counteracted in response to metformin during fast.

Acknowledgements We acknowledge the assistance of laboratory technicians B. Damby (University of Southern Denmark, Odense, Denmark), L. Hansen, C. B. Olsen, S. Hillbrandt, S. Feddersen and K. Skov (Odense University Hospital, Odense, Denmark). F. Alford (University of Melbourne, Melbourne, VIC, Australia) and R. Bergmann (Cedars-Sanai Diabetes and Obesity Research Institute, Los Angeles, CA, USA) are thanked for their contribution to the discussion. Some of the data were presented as an abstract at the 75th Scientific Sessions of the ADA, 2015 in Boston, USA.

Funding The study was supported by the Danish Research Council for Health and Disease (grant number 0602-02695B) and Odense University Hospital Free Research Fund in 2012.

Duality of interest The authors declare that there is no duality of interest associated with the manuscript.

Author contributions $\mathrm{MMHC}$ analysed and interpreted the data. MMHC, KH, PD, OH-N, KB and HB-N designed the study. TBS contributed to the data analysis. MMHC drafted the manuscript. All authors critically assessed and reviewed the manuscript. All authors gave final approval of the manuscript. MMHC is the guarantor of this work and takes responsibility for the data integrity. 


\section{References}

1. Inzucchi SE, Bergenstal RM, Buse JB et al (2012) Management of hyperglycaemia in type 2 diabetes: a patient-centered approach. Position statement of the American Diabetes Association (ADA) and the European Association for the Study of Diabetes (EASD). Diabetologia 55:1577-1596

2. Hundal RS, Krssak M, Dufour S et al (2000) Mechanism by which metformin reduces glucose production in type 2 diabetes. Diabetes 49:2063-2069

3. Hother-Nielsen O, Schmitz O, Andersen PH et al (1989) Metformin improves peripheral but not hepatic insulin action in obese patients with type II diabetes. Acta Endocrinol (Copenh) 120:257-265

4. Bailey CJ, Wilcock C, Scarpello JHB (2008) Metformin and the intestine. Diabetologia 51:1552-1553

5. Hirst JA, Farmer AJ, Ali R et al (2012) Quantifying the effect of metformin treatment and dose on glycemic control. Diabetes Care $35: 446-454$

6. Rena G, Pearson ER, Sakamoto K (2013) Molecular mechanism of action of metformin: old or new insights? Diabetologia 56:18981906

7. Miller RA, Chu Q, Xie J et al (2013) Biguanides suppress hepatic glucagon signaling by decreasing production of cyclic AMP. Nature 494:256-260

8. Madiraju AK, Erion DM, Rahimi Y et al (2014) Metformin suppresses gluconeogenesis by inhibiting mitochondrial glycerophosphate dehydrogenase. Nature 510:542-546

9. Gong L, Goswami S, Giacomini KM et al (2012) Metformin pathways: pharmacokinetics and pharmacodynamics. Pharmacogenet Genomics 22:820-827

10. Graham GG, Punt J, Arora M et al (2011) Clinical pharmacokinetics of metformin. Clin Pharmacokinet 50:81-98

11. Koepsell H (2004) Polyspecific organic cation transporters: their functions and interactions with drugs. Trends Pharmacol Sci 25: 375-381

12. Shu Y, Sheardown SA, Brown C et al (2007) Effect of genetic variation in the organic cation transporter 1 (OCT1) on metformin action. J Clin Invest 117:1422-1431

13. Shu Y, Brown C, Castro RA et al (2008) Effect of genetic variation in the organic cation transporter 1, OCT1, on metformin pharmacokinetics. Clin Pharmacol Ther 83:273-280

14. Christensen MMH, Brasch-Andersen C, Green H et al (2011) The pharmacogenetics of metformin and its impact on plasma metformin steady-state levels and glycosylated hemoglobin A1c. Pharmacogenet Genomics 21:837-850

15. Pedersen RS, Christensen MM, Brosen K (2012) Linkage disequilibrium between the CYP2C19*17 allele and other clinically important CYP2C allelic variants in a healthy Scandinavian population. Eur J Clin Pharmacol 68:1463-1464

16. Christensen MM, Hojlund K, Hother-Nielsen O et al (2015) Steady-state pharmacokinetics of metformin is independent of the octl genotype in healthy subjects. Eur J Clin Pharmacol 71:691697

17. Wahren J, Ekberg K (2007) Splanchnic regulation of glucose production. Annu Rev Nutr 27:329-345

18. Hother-Nielsen O, Henriksen JE, Holst JJ, Beck-Nielsen H (1996) Effects of insulin on glucose turnover rates in vivo: isotope dilution versus constant specific activity technique. Metabolism 45:82-91

19. Hother-Nielsen O, Mengel A, Moller J et al (1992) Assessment of glucose turnover rates in euglycaemic clamp studies using primedconstant [3-3H]-glucose infusion and labelled or unlabelled glucose infusates. Diabet Med 9:840-849

20. Frayn KN (1983) Calculation of substrate oxidation rates in vivo from gaseous exchange. J Appl Physiol Respir Environ Exerc Physiol 55:628-634
21. Holst JJ (1982) Evidence that enteroglucagon (II) is identical with the C-terminal sequence (residues 33-69) of glicentin. Biochem J 207:381-388

22. Staehr P, Hother-Nielsen O, Beck-Nielsen H et al (2007) Hepatic autoregulation: response of glucose production and gluconeogenesis to increased glycogenolysis. Am J Physiol Endocrinol Metab 292:E1265-E1269

23. Rossetti L, Lee YT, Ruiz J et al (1993) Quantitation of glycolysis and skeletal muscle glycogen synthesis in humans. Am J Physiol 265:E761-E769

24. Højlund K, Wildner-Christensen M, Eshøj O et al (2001) Reference intervals for glucose, $\beta$-cell polypeptides, and counterregulatory factors during prolonged fasting. Am J Physiol Endocrinol Metab 280:E50-E58

25. Becker K (1995) Principles and practice of endocrinology and metabolism, 2nd edn. Lippincott-Raven, Philadelphia

26. Cusi K, Consoli A, DeFronzo RA (1996) Metabolic effects of metformin on glucose and lactate metabolism in noninsulin-dependent diabetes mellitus. J Clin Endocrinol Metab 81:4059-4067

27. Unger RH, Aguilar-Parada E, Muller WA, Eisentraut AM (1970) Studies of pancreatic alpha cell function in normal and diabetic subjects. J Clin Invest 49:837-848

28. D'Alessio D (2011) The role of dysregulated glucagon secretion in type 2 diabetes. Diabetes Obes Metab 13(Suppl 1):S126-S132

29. DeFronzo RA (2009) From the triumvirate to the ominous octet: a new paradigm for the treatment of type 2 diabetes mellitus. Diabetes 58:773-795

30. Penhoat A, Fayard L, Stefanutti A et al (2014) Intestinal gluconeogenesis is crucial to maintain a physiological fasting glycemia in the absence of hepatic glucose production in mice. Metabolism 63: 104-111

31. Poretsky L (2010) Principles of diabetes mellitus. Springer Science \& Business Media, New York

32. Vidon N, Chaussade S, Noel M et al (1988) Metformin in the digestive tract. Diabetes Res Clin Pract 4:223-229

33. Bailey CJ (1995) Metformin and intestinal glucose handling. Diabetes Metab Rev 11(Suppl 1):S23-S32

34. Bonora E, Cigolini M, Bosello O et al (1984) Lack of effect of intravenous metformin on plasma concentrations of glucose, insulin, C-peptide, glucagon and growth hormone in non-diabetic subjects. Curr Med Res Opin 9:47-51

35. Stage TB, Brosen K, Christensen MM (2015) A comprehensive review of drug-drug interactions with metformin. Clin Pharmacokinet 54:811-824

36. Stage TB, Damkier P, Pedersen RS et al (2015) A twin study of the trough plasma steady-state concentration of metformin. Pharmacogenet Genomics 25:259-262

37. Zhou K, Donnelly L, Yang J et al (2014) Heritability of variation in glycaemic response to metformin: a genome-wide complex trait analysis. Lancet Diabetes Endocrinol 2:481-487

38. Tzvetkov MV, Saadatmand AR, Bokelmann K et al (2012) Effects of OCT1 polymorphisms on the cellular uptake, plasma concentrations and efficacy of the 5-HT(3) antagonists tropisetron and ondansetron. Pharmacogenomics J 12:22-29

39. Zhou K, Donnelly LA, Kimber CH et al (2009) Reduced-function SLC22A1 polymorphisms encoding organic cation transporter 1 and glycemic response to metformin: a GoDARTS study. Diabetes 58:1434-1439

40. Du BD, Du Bois EF (1989) A formula to estimate the approximate surface area if height and weight be known. 1916. Nutrition 5:303311

41. Levey AS, Bosch JP, Lewis JB et al (1999) A more accurate method to estimate glomerular filtration rate from serum creatinine: a new prediction equation. Modification of Diet in Renal Disease Study Group. Ann Intern Med 130:461-470 